\title{
Atypical post-kala-azar dermal leishmaniasis resembling histoid leprosy
}

\author{
A. CHAKRABARTI*, B. KUMAR* , A. DAS $\dagger \&$ \\ V. K. MAHAJAN* \\ * Department of Dermatology, Venereology and Leprology; $\dagger$ Depart- \\ ment of Morbid Anatomy, Postgraduate Institute of Medical Educa- \\ tion and Research, Chandigarh-160 012, India
}

Accepted for publication 11 February 1997

Summary An adult male with atypical lesions of post-kala-azar dermal leishmaniasis (PKDL) is described. He had extensive ulcerated noduloplaque lesions on his hands, feet and genitalia. He had been diagnosed and treated for leprosy in the past. He came from an area endemic for kala-azar and leprosy and had a previous history of kala-azar. There was an abundance of Leishman Donovan bodies in slitskin smears and in histopathology sections. There was a good therapeutic response to sodium stibogluconate. An ulcerative variant of PKDL has been described but is extremely rare. Extensive lesions with ulceration have not been described before to the best of our knowledge. The epidemiological significance of the case is discussed.

\section{Introduction}

Post-kala-azar dermal leishmaniasis (PKDL) is a complication of visceral leishmaniasis occurring 1-5 years after recovery from the original infection. ${ }^{1}$ The usual clinical presentation of hypopigmented macules and nodules are often a source of confusion with leprosy especially in areas where both the diseases are prevalent. Many people migrate to far-off places in pursuit of work, and one can be caught unawares, especially in areas where the disease is seen less often. This is particularly true if the presentation is atypical. Although a number of clinical variants of PKDL, including the ulcerative variety has been described, extensive ulceration has not yet been reported in literature. A case with extensive ulcerated noduloplaque lesions is presented here and the factors leading to initial misdiagnosis discussed. 


\section{Case report}

A 35-yr-old man from Bihar (an endemic area for kala-azar and leprosy) working as a mason in Chandigarh (a city in the north-western part of India in which kala-azar is nonendemic) had had ulcerated plaque lesions over his hands and feet for 3 years. The lesions started over the dorsa of feet as asymptomatic papules which slowly increased in size and ulcerated in the centre. Over a period of 3 years similar ulcerated plaques appeared over the dorsa of the hands, scrotum and penis. He gave a history of hypoaesthesia over the extremities. He had consulted numerous doctors and had been consistently diagnosed as a case of leprosy and took treatment irregularly. He was subsequently referred to us for management.

On examination he was well built, nonicteric with no pallor. There was no significant lymphadenopathy except for 2-3 firm, discrete mobile nodes in the axilla about $1 \mathrm{~cm}$ in diameter. There was no hepatosplenomegaly.

There were firm, nontender skin-coloured plaques of varying sizes over the interphalangeal joints of the fingers, toes, feet (Figure 1) scrotum and glans penis (Figure 2). Most of the plaques were ulcerated and had some crusting. Scarring was evident in some plaques. A hypopigmented patch about $6 \times 8 \mathrm{~cm}$ in size was noted on the left shoulder. Its margins were irregular but well defined. On testing for sensation, an inconsistent response was noted as regards the degree and extent of loss over both the patch and the extremities. There was an interobserver variation as far as the peripheral nerves were concerned with some insisting that there was nerve thickening on the right side. All mucosae other than the glans were free of lesions.

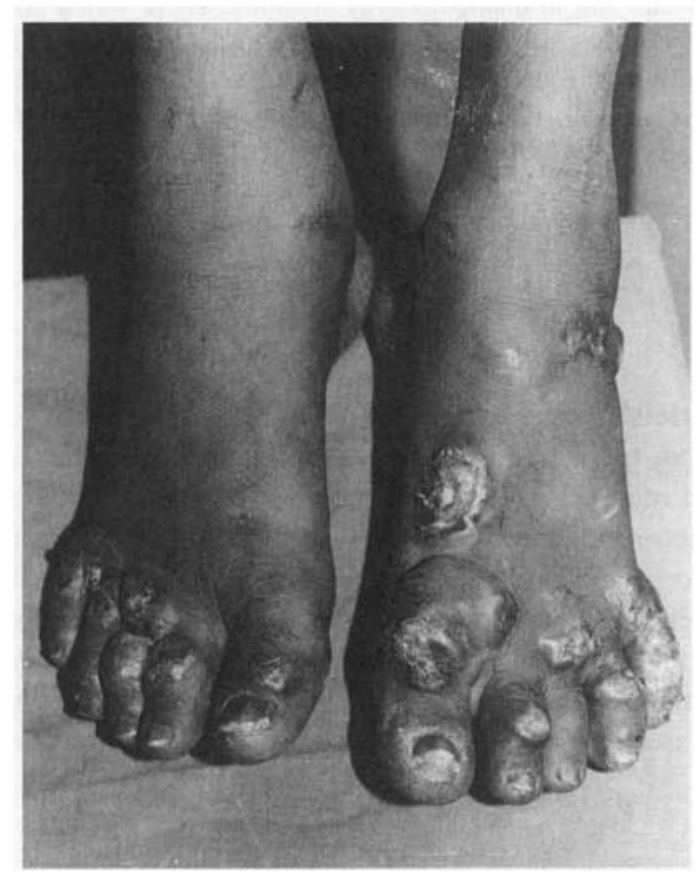

Figure 1. Ulcerated nodulo-plaque lesions on feet. 


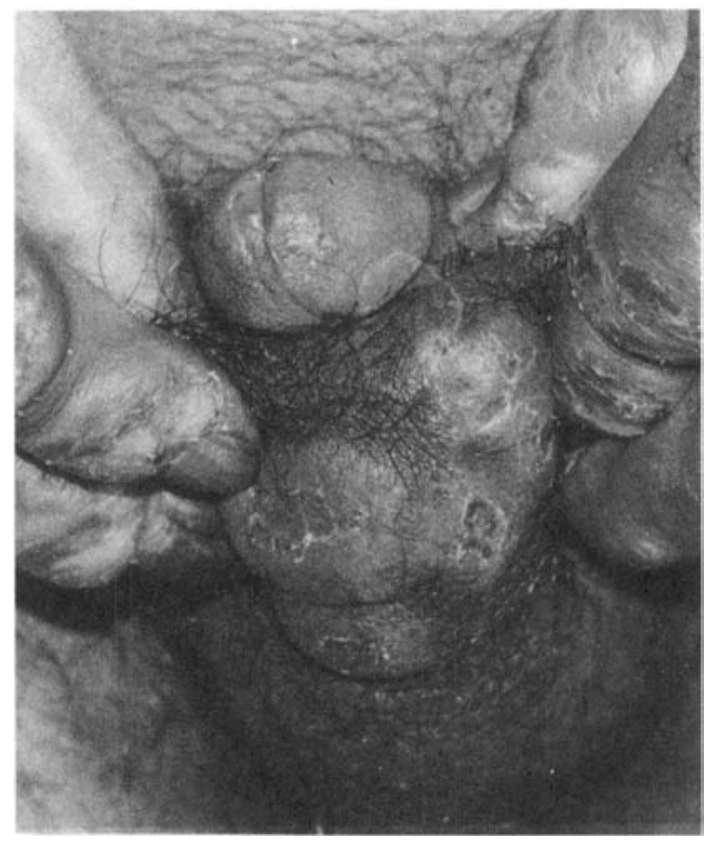

Figure 2. Ulcerated nodulo-plaque lesions on hands, scrotum and glans penis.

A provisional diagnosis of subpolar lepromatous leprosy with either lepromas or histoid lesions was made and the patient was further investigated.

Routine examination of blood and urine including haemogram, liver and renal function tests were normal except for minimal hypergammaglobulinaemia on electrophoresis. The chest X-Ray and ECG were also normal. Repeated slit-skin smear examination from routine and lesional sites were negative for acid-fast bacilli. On staining with Giemsa an abundance of Leishman Donovan (LD) bodies was seen in smears from the plaques. LD bodies though scanty were also noted in the smears from the hypopigmented patch. On histopathology, dense dermal infiltrate of plasma cells, lymphocytes and few neutrophils with a clear Grenz zone and numerous LD bodies were seen (Figure 3). The hypopigmented patch showed epitheloid granulomas in the dermis alongwith LD bodies. A biopsy from the right radial cutaneous nerve was of normal morphology and no organisms were seen even with special stains. Fine needle aspiration of the lymphnodes showed reactive lymphoid tissue and no organisms. Bone marrow trephine aspirate showed normocellular marrow with adequate representation of all cell lines. No LD bodies were seen.

Culture in NNN medium was positive from the plaques and hypopigmented patch. Neither the bone marrow aspirate nor the nerve specimen grew leishmania on culture. When questioned the patient admitted to having kala-azar about 10 years ago when he was probably treated with sodium stibogluconate for 18 days.

With a diagnosis of PKDL the patient was started on sodium stibogluconate injected $20 \mathrm{mg} / \mathrm{kg} / \mathrm{day}$ with a remarkable improvement. At the end of 2 months of therapy the plaques had flattened considerably and all the ulcers had healed. He received stibogluconate for another 2 months at a dose of $750 \mathrm{mg}$ every alternate day as he was unable to tolerate the pain 


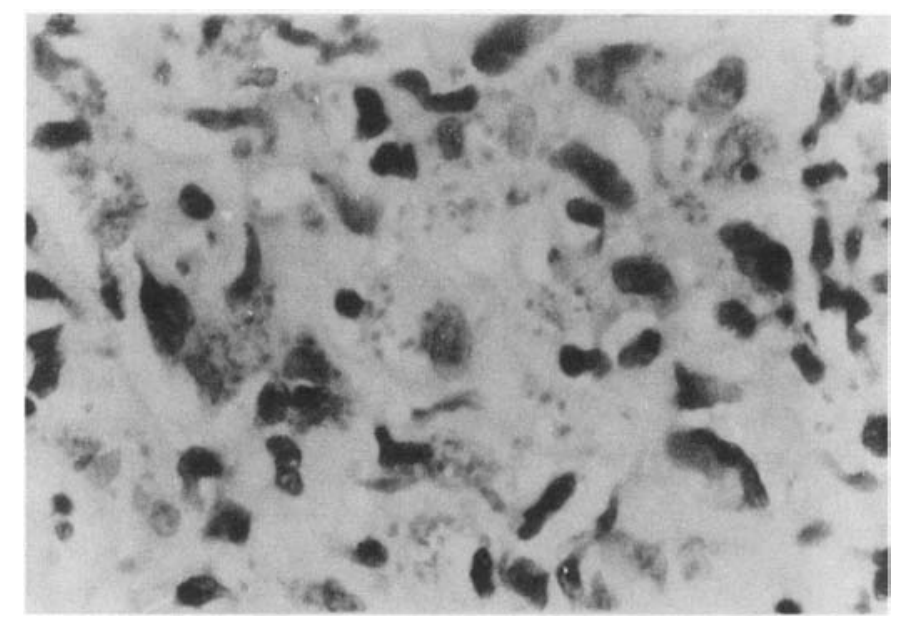

Figure 3. Numerous LD bodies in dermal infiltrate from nodule. $(H \& E, \times 240)$.

and discomfort at the injection site. After 4 months of therapy all plaques had flattened and the treatment was stopped. He is regularly followed up.

\section{Discussion}

PKDL can have varied manifestations, the commoner presentations being the hypopigmented lesions, erythema or butterfly rashes and nodules. ${ }^{1}$ The other less common variants are the verrucous, papillomatous, hypertrophic and xanthomatous lesions. Periungual induration, extensive erythema and fibroid types have also been described. ${ }^{2}$

Ever since the first description by Brahmachari in $1922,{ }^{3}$ PKDL has been frequently misdiagnosed as leprosy. The findings of a large hypopigmented patch and bilateral symmetrical ulcerated nodulo plaques over the hands, feet, scrotum and penis coupled with the history, albeit vague of hypoaesthesia over the lesions as well as in a glove and stocking distribution misled us. The hypopigmented lesions in PKDL generally do not attain a size larger than $1 \mathrm{~cm}$ but larger lesions, even occupying an entire limb, are well described. ${ }^{1}$ Ulceration in lesions of PKDL is extremely rare. It has been described after treatment with potassium iodide, trauma ${ }^{4}$ and mucosal stretching with granuloma. ${ }^{6}$ Though trauma can partly explain ulceration over the hands and feet related to his job, it cannot explain ulceration over the scrotum and glans. An ulcerative variant of the nodular type has been described by Smith \& Halder $^{2}$ but in all the three cases described by them the ulcerated nodules were limited in distribution. However, the extensive ulceration seen in our patient, to the best of our knowledge, has not been described before.

Neuritis has been described in cutaneous lesions of $\mathrm{PKDL}^{7}$ but peripheral neuritis is not known. In our opinion repeated questioning about lesional and peripheral sensory loss resulted in autosuggestion.

Refractoriness of PKDL to therapy and the fact that parasitic load may determine the response to therapy is well known. ${ }^{8}$ Though to date the response in the patient has been 
excellent, it remains to be seen how the disease behaves on follow up. The response to therapy of this variant and the long-term prognosis is not known due to paucity of such cases.

This report exemplifies the pitfalls of diagnosis made only on basis of history and clinical examination. PKDL should always be considered in differential diagnosis of any patient from an endemic area for kala-azar, however atypical the lesions might be.

\section{References}

${ }^{1}$ Napier LE and Dasgupta CR. A clinical sutdy of post kala azar dermal leishmaniasis. Ind Med Gaz 1930; 65: 249-257.

2 Smith ROA and Halder KC. Some observation on dermal leishmaniasis. Ind Med Gaz 1935; 70: 544-550.

${ }^{3}$ Brahmachari UN. A new form of cutaneous leishmaniasis. Ind Med Gaz 1922; 57: 128.

${ }^{4}$ Napier LE. Kala azar In: The principles and practice of tropical medicine. New York: Macmillan, 1946: 135.

5 Ramesh V, Saxena U, Misra RS and Mukherjee A. Post kala-azar dermal leishmaniasis: a case report strikingly resembling lepromatous leprosy. Lepr Rev 1991; 62: 217-221.

${ }^{6}$ Napier LE and Dasgupta CR. Further clinical observation on dermal leishmaniasis. Ind Med Gaz 1934; 69: 121-130.

7 El Hassan AM, Ghalib HW, Zijlotra EE et al. Post kala-azar dermal leishmaniasis in Sudan: clinical features, pathology and treatment. Trans R Soc Trop Med Hyg 1992; 86: 245-248.

8 Erocolli N. Drug responsiveness in experimental cutaneous leishmaniasis. Exp parasit 1966; 19: 320.

9 Girgla HS, Marsden RA, Singh GM and Ryan TJ. Post kala-azar dermal leishmaniasis. Br J Dermatol 1977; 97: 307-311. 